\title{
Agentenbasiertes Planungsmodell für die Grobplanung von Kommissioniersystemen
}

\author{
Agent based planning model for the plan of concept for order picking systems
}

\author{
Marita Ellinger \\ Michael ten Hompel \\ Fraunhofer-Institut für Materialfluss und Logistik IML, Dortmund
}

$\mathbf{R}$ echnergestützte Modellansätze, die Logistiksysteme gestalten und generieren, sind eine hochkomplexe Aufgabenstellung. Die bisher in der Praxis existierenden Planungs- und Steuerungsmodelle für Intralogistiksysteme weisen für die aktuellen und zukünftigen Anforderungen wie der Komplexitätsbewältigung, Reaktionsschnelligkeit und Anpassungsfähigkeit Schwachstellen auf. - Ein innovativer Ansatz, diesen Ansprüchen gerecht zu werden, stellen Multiagentensysteme dar. Mit ihrem dezentralen und modularen Charakter sind sie für ein komplexes Problem mit einem geringen Grad an Strukturiertheit geeignet. Außerdem ermöglichen diese computergestützten intelligenten Systeme den Anwendern eine einfache und aufwandsarme Handhabung.

[Schlüsselwörter: Multiagentensystem, Kommissioniersystem, Planungsmodell, automatisierte Planung, PASSI-Methode]

C omputer aided systems for designing and generating logistic systems are due to the complexity of the task very difficult to build. The existing planning and control models for inhouse logistic systems are not fulfilling the modern requirements on coping with the complexity, speed of reaction and flexibility. - The use of multi agent systems is one innovative approach to fulfil those requirements. Because of their decentralized and modular characteristic, they are very useful for solving ill-structured and complex problems. Furthermore, these intelligent computer aided systems make an easy and effortless use possible.

[Keywords: multi agent system; order picking system, planning model; automated planning, PASSI-Method]

\section{Einleitung}

Ein Umdenken in der Entwicklung und Nutzung intralogistischer Planungsmodelle muss stattfinden. Den zunehmend komplexer werdenden Strukturen in der Logistik sollte nicht mit kompliziert anzuwendenden Planungsmodellen begegnet werden. Gefragt ist im Gegenteil ein automatisiertes Modell, welches einen sinnvollen Umgang mit der hohen Komplexität der Planungsaufgabe ohne die Notwendigkeit von ausgeprägtem Erfahrungswissen ermöglicht. Diese Forderung sollte gerade für die Konzeptplanung von Kommissioniersystemen berücksichtigt werden. Die Entwicklung von Grobkonzepten für Kommissioniersysteme gehört zu den schwierigsten Aufgaben in der Intralogistik [HSB11].

In der Praxis ist allerdings das Gegenteil der Fall. Bestehende Planungsmodelle weisen eine unzureichende Unterstützung bei dem Design des neuen Kommissioniersystems auf [AMW10]. Das Systemdesign beinhaltet in diesem Zusammenhang die Auswahl und die Dimensionierung von Systemkomponenten und deren Verknüpfung zu einem Gesamtsystem. Bei der Technikauswahl zur Systemgestaltung und wesentlichen Fragestellungen, (z.B. ob ein einstufiges oder ein zweistufiges System zu bevorzugen ist), ist der Planende auf sein Erfahrungswissen angewiesen.

Die bestehenden Planungsmodelle verfolgen den Ansatz einer hierarchischen oder zentralen top-down- Organisation (vgl. z.B. [JD89]). Während der Konzeptplanung für ein Kommissioniersystem muss eine Vielzahl von Technikkomponenten und technischen sowie organisatorischen Realisierungsmöglichkeiten ausgewählt und bewertet werden. Zudem sind eine Vielzahl von Daten, Restriktionen, Prozessspezifika, etc. zu verarbeiten [HSB11]. Dies macht diese Aufgabe so umfangreich und schwierig.

In Anbetracht dieser Tatsache stellt sich die Frage, ob die beschriebene Komplexität mit dem bestehenden Ansatz abzubilden oder ob nicht ein anderer Ansatz zu bevorzugen ist. Für hochkomplexe Problemstellungen werden in anderen Fachgebieten seit Jahren Agentensysteme eingesetzt. Agentenbasierte Systeme haben durch ihre dezentrale Struktur großes Potenzial bei der Lösung komplexer Aufgabenstellungen gezeigt [Par98]. Diese Leistungsfähigkeit von dezentral organisierten Systemen wurde vor einigen Jahren für die Steuerung logistischer Systeme erschlossen [GH10]. In einigen Forschungsprojekten wurden bereits agentenbasierte Systeme, bspw. zur Materialflusssteuerung [LCL10], entwickelt. 
Für die Planung von Intralogistiksystemen im Allgemeinen und für Kommissioniersysteme im Speziellen wurde dieser Ansatz bisher nicht eingesetzt. Diese Lücke soll zukünftig durch die Forschung am Fraunhofer-Institut für Materialfluss und Logistik IML in Dortmund geschlossen werden, an dem zurzeit eine Forschungsinitiative zu diesem Thema gestartet wird. Ein Teil dieser Forschungsinitiative ist die Entwicklung eines agentenbasierten Planungsmodells für die Grobplanung von Kommissioniersystemen, welche in diesem Beitrag betrachtet wird.

Dieser Beitrag beinhaltet folgende vier Punkte: Zunächst wird der Hintergrund des hier vorgestellten Planungsmodells dargestellt. Danach werden die Vorgehensweise und die Methode zur Entwicklung des Agentensystems vorgestellt. Schließlich werden die funktionalen Anforderungen an das Planungssystem beschrieben. Am Ende des Beitrages wird ein Ausblick auf die zukünftig folgenden Entwicklungsschritte gegeben.

\section{STAND DER FORSCHUNG}

In diesem Abschnitt werden der Stand der Forschung und die Problemstellung erläutert, um daraus die Forschungslücke und das Ziel der Forschungstätigkeit abzuleiten.

\subsection{KOMMISSIONIERSYSTEMPLANUNG}

Die Planung von Kommissioniersystemen, insbesondere das Systemdesign, ist auch heute noch kein standardisierter Prozess [Ulb10]. Sie beruht vielmehr auf den Erfahrungen, dem Wissen und dem Hintergrund des Planers. Die Planungsentscheidungen sind folglich subjektiv geprägt und häufig nicht transparent und nachvollziehbar [JNS09]. Dies führt dazu, dass nicht unbedingt immer das Kommissioniersystem realisiert wird, welches die gegebenen und zukünftigen Anforderungen optimal erfüllt.

Ein ungeeignetes Kommissioniersystem hat große Auswirkungen auf die Wettbewerbsfähigkeit eines Unternehmens. Das Kommissioniersystem verursacht einen großen Anteil der Logistikkosten eines Unternehmens [Gud10]. Die Planung selbst ist in der Regel mit einem hohen Aufwand verbunden [GGU09], wodurch auch hier hohe Kosten entstehen können. Denn die Bewältigung dieser schwierigen Aufgabe ist derzeit aufgrund einer mangelnden technischen und theoretischen Unterstützung den Experten der Branche vorbehalten. Bedenkt man, dass die Nutzungsdauer eines Kommissioniersystems aufgrund der Dynamik der Märkte stetig sinkt und sich somit die Planungsintervalle weiter verkürzen, rückt die Senkung der Planungsdauer und damit der Planungskosten in den Fokus [JT12].

Die drei wesentlichen Ursachen für die beschriebenen Defizite liegen in folgenden Punkten: Die existierenden Planungsvorgehensmodelle sind zu abstrakt, als dass sie den Planer während des Planungsprozesses mit kon- kreten Hinweisen und Entscheidungsregeln unterstützen könnten. Die Arbeiten zum Systemdesign sind zu theoretisch, um als praktische Anwendung zu dienen oder sie konzentrieren sich auf einen klar abgegrenzten Detailbereich der Planungsaufgabe. Drittens fehlt eine umfassende und nutzerfreundliche Computerunterstützung während des Systemdesigns. Diese Ursachen werden nachfolgend näher erläutert.

\section{Planungsvorgehensmodelle}

Bestehende Planungsvorgehensmodelle (bspw. [JD89]) sind charakterisiert durch hierarchisch aufeinanderfolgende, iterativ zu durchlaufende, abgeschlossene Schritte [JNS09]. Diese Schritte bieten dem Planer einen groben Ablaufplan für die Bewältigung der Planungsaufgabe, sie geben jedoch keine konkreten Hinweise für die Durchführung des Planungsprozesses oder auf Entscheidungsregeln und -grundlagen. Da diese Vorgehensmodelle allgemeingültig formuliert sind, müssen sie zwangsläufig vom konkreten Anwendungsfall abstrahieren. Dadurch verlieren sie allerdings für den konkreten, praktischen Planungsfall an Nutzen.

\section{Arbeiten zum Systemdesign}

Die Arbeiten zur Systemauswahl und -dimensionierung sind in der Regel durch eine Reduktion der Komplexität gekennzeichnet. Die Mehrheit dieser Arbeiten basiert auf der Strukturierung und Klassifizierung von Kommissioniersystemen (bspw. [Töp95]), welche in der VDIRichtlinie 3590 dargestellt ist [VDI3590]. Dort wird das Kommissioniersystem in ein Materialflusssystem, ein Informationsflusssystem und die Organisation unterteilt. Viele Arbeiten zum Systemdesign beschränken sich auf einen dieser drei Teilbereiche (bspw. [Pot94]). Andere Arbeiten sind fokussiert auf eine Detailproblemstellung während der Kommissioniersystemplanung (z.B. [Sad07]).

In der jüngeren Vergangenheit wurde von der in der VDI-Richtlinie 3590 enthaltenen Strukturierung abgewichen. Unter anderem wurden für die Anwendung in der Simulation Standardkommissionierbausteine entwickelt (bspw. [MM07]). Diese Bausteine abstrahieren erneut von der Realität (sie bilden bspw. nicht alle bestehenden Kommissioniersysteme ab) und liefern dem Anwender wiederum keine Hilfestellung bei einer konkreten, praktischen Planungsaufgabe. Es kann bezweifelt werden, dass die Entwicklung von Standardkommissionierbausteinen für die Unterstützung während einer konkreten Planungsaufgabe Erfolg versprechend ist. Im Bereich der Steuerung logistischer Systeme ließ sich bereits feststellen, dass die Entwicklung von Standardprozessketten nicht zielführend ist [Hom08].

\section{Fehlende Computerunterstützung}

Die Schwierigkeit der Planungsaufgabe und der mit der Planung verbundene Zeit- und Kostenaufwand legt ei- 
ne Computerunterstützung nahe. Nun stellt sich die Frage, warum es diese bisher nur in geringem Maße gibt [JT12]. Dies liegt im Wesentlichen in der Komplexität der Planungsaufgabe, der Art der Problemstellung und den eingesetzten Methoden begründet.

Eine Unterstützung des Planers im konkreten Anwendungsfall gelingt erst, wenn die Komplexität der Planungsaufgabe nicht reduziert, sondern abgebildet wird. Trotz der Abbildung darf der Anwender nicht durch eine schwierige Bedienung oder die aufwändige Anwendung der entwickelten Modelle und Verfahren überfordert werden. Die Problemstellung zeichnet sich durch einen geringen Grad der Strukturierung aus [Sch08]. Aus diesem Grund sind viele eingesetzte Methoden, wie analytische oder statistische Methoden, nicht oder nur eingeschränkt für diese Problemstellung anwendbar.

Zusammenfassend lässt sich festhalten, dass die Kommissioniersystemplanung weiterhin ein personengetriebener Prozess mit geringer Computerunterstützung ist. Die Ergebnisqualität hängt im Wesentlichen von der planenden Person ab. Ein möglicher Ansatz, um diese Lücke zu schließen, sind Agentensysteme, welche sich in anderen Bereichen der Logistik bereits als sehr wirkungsvoll erwiesen haben.

\subsection{AgENTENSYSTEME IN DER LOGISTIK}

Multiagentensysteme werden in der Logistik derzeit überwiegend für die Steuerung eingesetzt. Untersuchte Einsatzgebiete reichen dabei vom Supply Chain Management über die Transportlogistik bis zur Intralogistik. In der operativen Supply Chain Planung wurde z.B. ein Modell zur Integration von agentenbasierter Logistik- und Produktionsplanung entwickelt [HB10]. Die operative Planung und Terminierung ist $\mathrm{u}$. a. Gegenstand von agentenbasierten Lösungen in der Transportlogistik [DHR+05]. In der Intralogistik werden bspw. ein Agentensystem für die Materialflussteuerung [Lib11] und die Steuerung von zellularer Fördertechnik mit Multiagentensystemen untersucht [KSN+11].

Die Erforschung des Einsatzes von Agentensystemen für die gestalterische Planung logistischer Systeme steht bisher noch aus. In artverwandten Bereichen, wie der Fabrikplanung, sind jedoch erste Ansätze in diese Richtung getätigt worden (bspw. [FO05]).

\subsection{ZIELSTELLUNG}

Das Ziel der Entwicklung eines agentenbasierten Planungsmodells ist die Unterstützung des Nutzers in konkreten Anwendungsfällen durch ein computergestütztes Planungssystem. Das System soll für die vom Anwender eingegebenen Anforderungen Lösungsalternativen generieren und diese dem Planer inkl. der jeweiligen Systemkennwerte ausgeben. Der Vergleich und die Bewer- tung der Systeme erfolgt weiterhin anhand der gelieferten Kennzahlen durch den Planer. Dieser kann so für die abschließende Entscheidung qualitative Kriterien hinzuziehen, welche das Planungssystem nicht abbilden kann.

Die Automatisierung der Systemkonzeption soll die Planungsdauer deutlich verkürzen, die Kosten für die Planung senken und die Transparenz und Objektivität der Planungsergebnisse steigern. Veränderungen, bspw. in der Ausgangssituation, sollen schnell und leicht berücksichtigt und die Auswirkung dieser Veränderungen analysiert werden können. Darüber hinaus soll das Planungssystem wandlungsfähig sein. Damit können neue Techniken oder detailliertere Berechnungsverfahren mit geringem Aufwand integriert werden.

\section{VORGEHENSWEISE ZUR SYSTEMENTWICKLUNG}

Für die Entwicklung des agentenbasierten Planungssystems wurde die PASSI (Process for Agent Societies Specification and Implementation) -Methode ausgewählt. Sie stammt aus der agentenorientierten Softwareentwicklung und beinhaltet eine strukturierte Entwicklungsvorgehensweise [CP02]. Diese bietet zum einen den Vorteil, dass sie den Entwickler im Softwareentwicklungsprozess schrittweise von der Anforderungsanalyse bis zur Implementierung unterstützt [Lib11]. Zum anderen nutzt sie zur Visualisierung die UML (Unified Modeling Language), einen Standard in der Softwareentwicklung [Oes06].

Die PASSI-Methode setzt sich, wie in Abbildung 1 dargestellt, aus fünf Phasen zusammen, die iterativ durchlaufen werden. Die Entwicklungsstufen beinhalten folgende Schwerpunkte [CP02]:

Anforderungsmodell (System Requirements Model): In dieser Entwicklungsphase werden die funktionalen Anforderungen an das System analysiert.

Agentenmodell (Agent Society Model): Die in der ersten Phase evaluierte erste Lösung wird in der zweiten Phase weiter spezifiziert. Hierzu werden die Interaktionen und die Abhängigkeiten zwischen den Agenten identifiziert und beschrieben.

Implementierungsmodell (Agent Implementation Model): Nach diesen beiden Entwicklungsphasen wird eine implementierungsnahe Beschreibung der Agenten selbst und des Multiagentensystems entwickelt.

Code-Modell (Code Model): Die entwickelten Modelle werden durch CASE (computer aided software engineering)-Werkzeuge in Code-Schablonen umgesetzt.

Einsatzmodell (Deployment Model): Abschließend wird im Einsatzmodell die Verteilung der Software auf die verfügbare Hardware spezifiziert. 


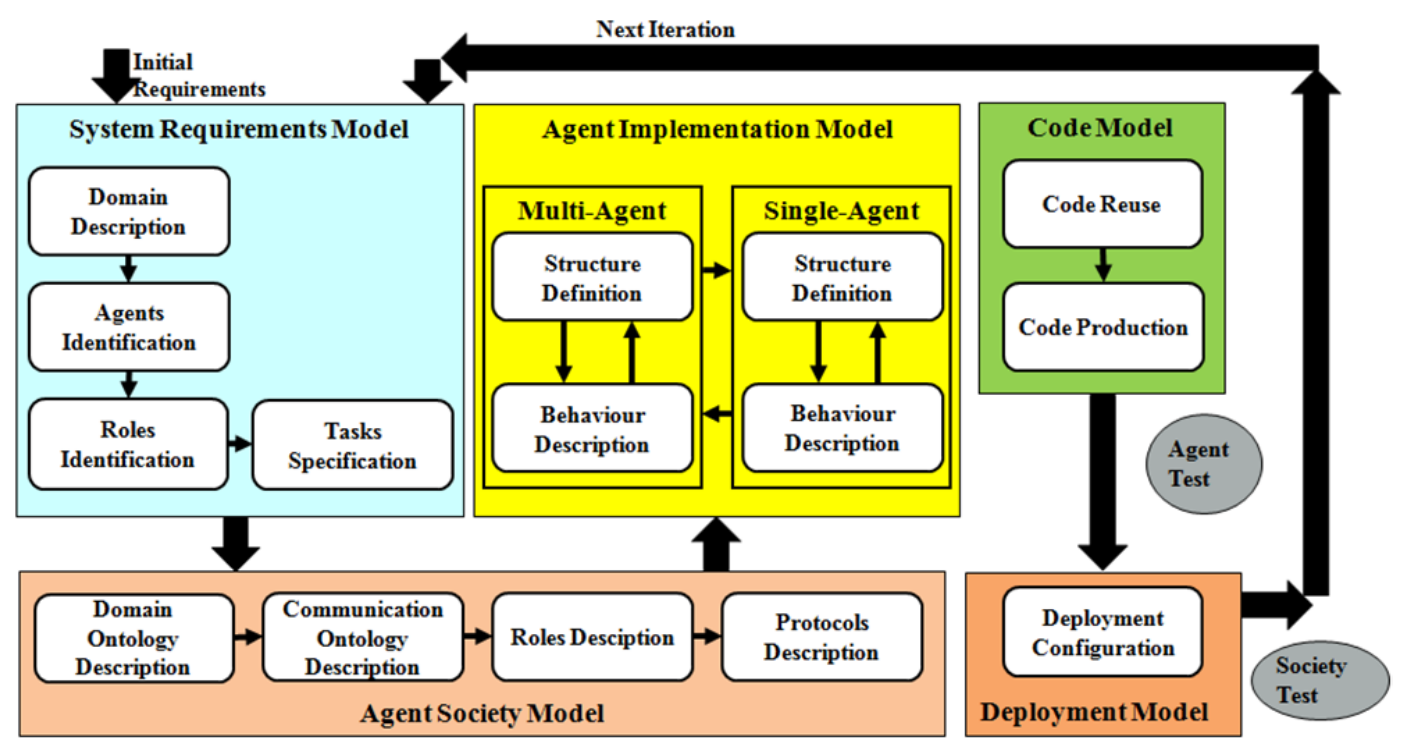

Abbildung 1. Phasen der PASSI-Methode [CCSO4]

\section{DOMÄNENBESCHREIBUNG (DOMAIN DESCRIPTION)}

Die Entwicklung des Anforderungsmodells ist der erste Schritt in der agentenbasierten Softwareentwicklung. Dieses Modell beinhaltet die Identifikation und Beschreibung sowohl der funktionalen als auch der nichtfunktionalen Anforderungen an das agentenbasierte Planungssystem.

\section{Nichtfunktionale Anforderungen}

Die nichtfunktionalen Anforderungen beziehen sich auf Faktoren wie Benutzerfreundlichkeit, Benutzeroberfläche oder Wartung und Pflege des Systems. Die Identifikation dieser Anforderungen wird von der PASSIMethode nicht unterstützt.

In diesem Beitrag werden die nichtfunktionalen Anforderungen nicht betrachtet. Zwar sind nichtfunktionale Anforderungen für die Nutzung und Akzeptanz des Planungssystems von großer Bedeutung, sie sind jedoch nicht essentiell für die Darstellung der Funktionsweise des Systems.

\section{Funktionale Anforderungen}

Funktionale Anforderungen spiegeln alle erforderlichen Funktionalitäten des Systems wider. So muss bspw. das hier vorgestellte Planungssystem die Eingabe von Restriktionen oder die Auswahl der Ein-/Auslagertechnik unterstützen. Mit der PASSI-Methode werden die funktionalen Anforderungen, analog zur objektorientierten Analyse [CP02] durch die Evaluation von Anwendungsfällen (engl. Use Cases) ermittelt. Die Darstellung dieser funktionalen Anforderungen ist für das Systemverständnis entscheidend. Deshalb wird dieser Entwicklungsschritt im folgenden Abschnitt detailliert erläutert.

\subsection{DEFINITION DER SYSTEMUMGEBUNG}

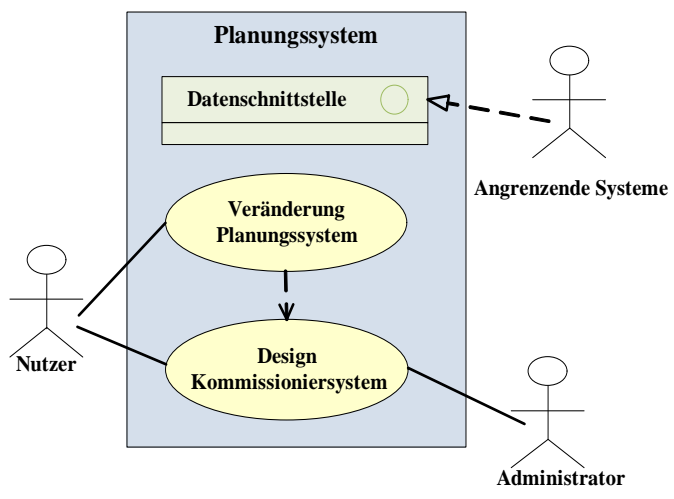

Abbildung 2. Kontextdiagramm

Die Anwendungsfälle werden vom Groben zum Feinen entwickelt und detailliert. Daraus resultiert eine hierarchische Anordnung von Anwendungsfällen. Diese Vorgehensweise ist aus der objektorientierten Analyse bekannt [Oes06].

Die Anforderungsanalyse beginnt mit der Darstellung der Systemschnittstellen in einem Kontextdiagramm (siehe Abbildung 2). In diesem Diagramm sind die beiden allgemeinen Anwendungsfälle Design Kommissioniersystem und Veränderung Planungssystem und die mit dem System interagierenden Akteure abgebildet.

\section{Die Anwendungsfälle}

Der Anwendungsfall Design Kommissioniersystem beinhaltet alle Funktionalitäten, welche für die Grobplanung eines Kommissioniersystems erforderlich sind, wie die Interaktion mit dem Nutzer zur Eingabe von Daten oder die Berechnung der Personalkosten durch das System. Diese Funktionen sind in weiteren Anwendungsfällen detaillierter auszuarbeiten. Aus diesen detaillierteren 
Betrachtungen entstehen sog. Domänenbeschreibungsdiagramme, die in Kapitel 4.2 näher erläutert werden.

Die Wandlungsfähigkeit und Robustheit ist aktuell und zukünftig ein wesentlicher Faktor für die Qualität eines Systems oder Modells. Aus diesem Grund wurde für die Wartung, für die Erweiterung und für die Anpassung des Systems ein eigenständiger Anwendungsfall integriert: Veränderung Planungssystem. Die Anwendbarkeit des zu entwickelnden Systems basiert u.a. auf aufwandsarmer Erweiterbarkeit und Austauschbarkeit von Teilen des Planungssystems. Allerdings konzentriert sich dieser Beitrag auf die Darstellung der Planungsfunktionalitäten. Deshalb wird der Anwendungsfall Veränderung Planungssystem nachfolgend nicht weiter betrachtet.

\section{Die Akteure}

Die Nutzer dieses Planungssystems sind Logistikunternehmen, Planungsunternehmen und Technikanbieter. Diese drei Nutzergruppen unterscheiden sich hinsichtlich des Einsatzzweckes und des Umfangs des Planungssystems. Das Logistikunternehmen wird in der Regel das Planungssystem nutzen, um für das eigene Unternehmen ein neues Kommissioniersystem zu planen. Ein Planungsunternehmen wird dagegen weniger für sein eigenes Unternehmen, als vielmehr für andere Unternehmen die
Kommissioniersysteme planen. Dies erfordert bspw. die Nutzung des Planungssystems auf unterschiedlichen Computern zur gleichen Zeit. Ein Technikanbieter wird ebenso wie ein Planungsunternehmen in der Regel für andere Unternehmen planen, jedoch wahrscheinlich mit dem eigenen Portfolio an Technikkomponenten. Dies erfordert eine Individualisierung des Planungssystems.

Der Administrator verfügt über das Recht, das Planungssystem zu verändern. Da dieser Anwendungsfall nicht der Fokus dieses Beitrages ist, soll hier nur kurz anhand eines Beispiels auf die Aufgaben eines Administrators eingegangen werden. Mit einer Ausbauphase dieses Planungssystems wird es möglich sein, dass die Technikanbieter ihre Technikkomponenten und ihre Planungsvorgehensweise in diesem System abbilden lassen können. Die Integration dieser Funktionalitäten obliegt dann dem Administrator.

Angrenzende Systeme sind insbesondere Verwaltungssysteme wie ein ERP, ein WMS oder ein MFR. Es wird die Möglichkeit berücksichtigt, dass in einer weiterentwickelten Form dieses Planungssystems die erforderlichen Daten nicht mehr durch die Eingabe des Nutzers bereitgestellt werden. Das Planungssystem erhält sie direkt aus den angrenzenden Systemen, um sie daraufhin automatisch auszuwerten.

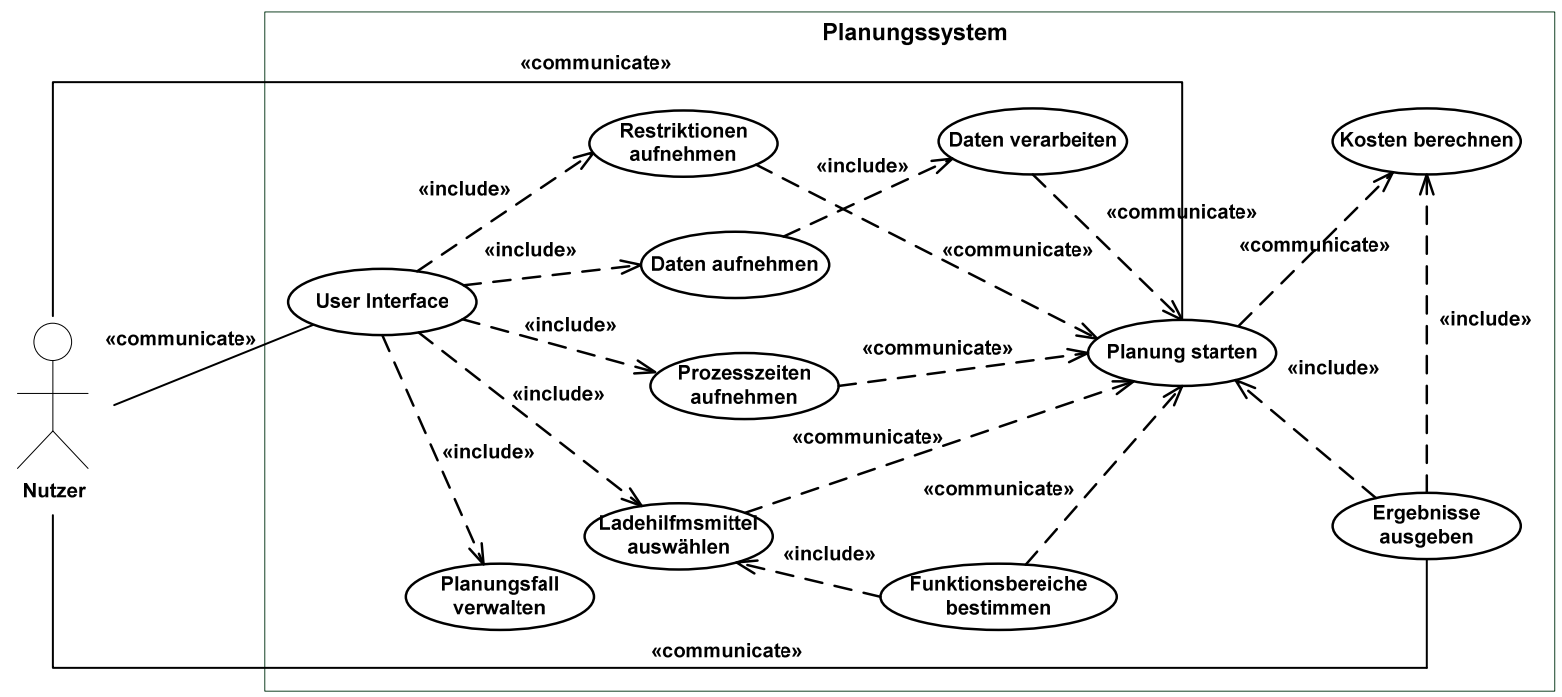

Abbildung 3. Domänenbeschreibungsdiagramm Vorbereitungsfunktionen

\section{2 ÜBERGEORDNETE DOMÄNENBESCHREIBUNGSDIAGRAMME}

Die Detaillierung des Anwendungsfalls Design Kommissioniersystem wird nachfolgend beschrieben. Aus Gründen der Übersicht sind in diesem Fall zwei Domänenbeschreibungsdiagramme erstellt worden. Sie spiegeln unterschiedliche Betrachtungswinkel auf das Planungssystem wider: Die Interaktion mit dem Nutzer und die syste- mische Planung. Beide Diagramme zusammen stellen die Anforderungen an das agentenbasierte Planungssystem dar.

In der Abbildung 3 ist das Domänenbeschreibungsdiagramm dargestellt, das die Interaktion des Systems mit dem Nutzer in den Vordergrund rückt. Hier sind die Systemfunktionen beschrieben, die der eigentlichen systematischen Planung vorgeschaltet sind. Beispielsweise werden hier durch das System Restriktionen, Daten, Informationen zum Kommissionierprozess etc. abgefragt. 


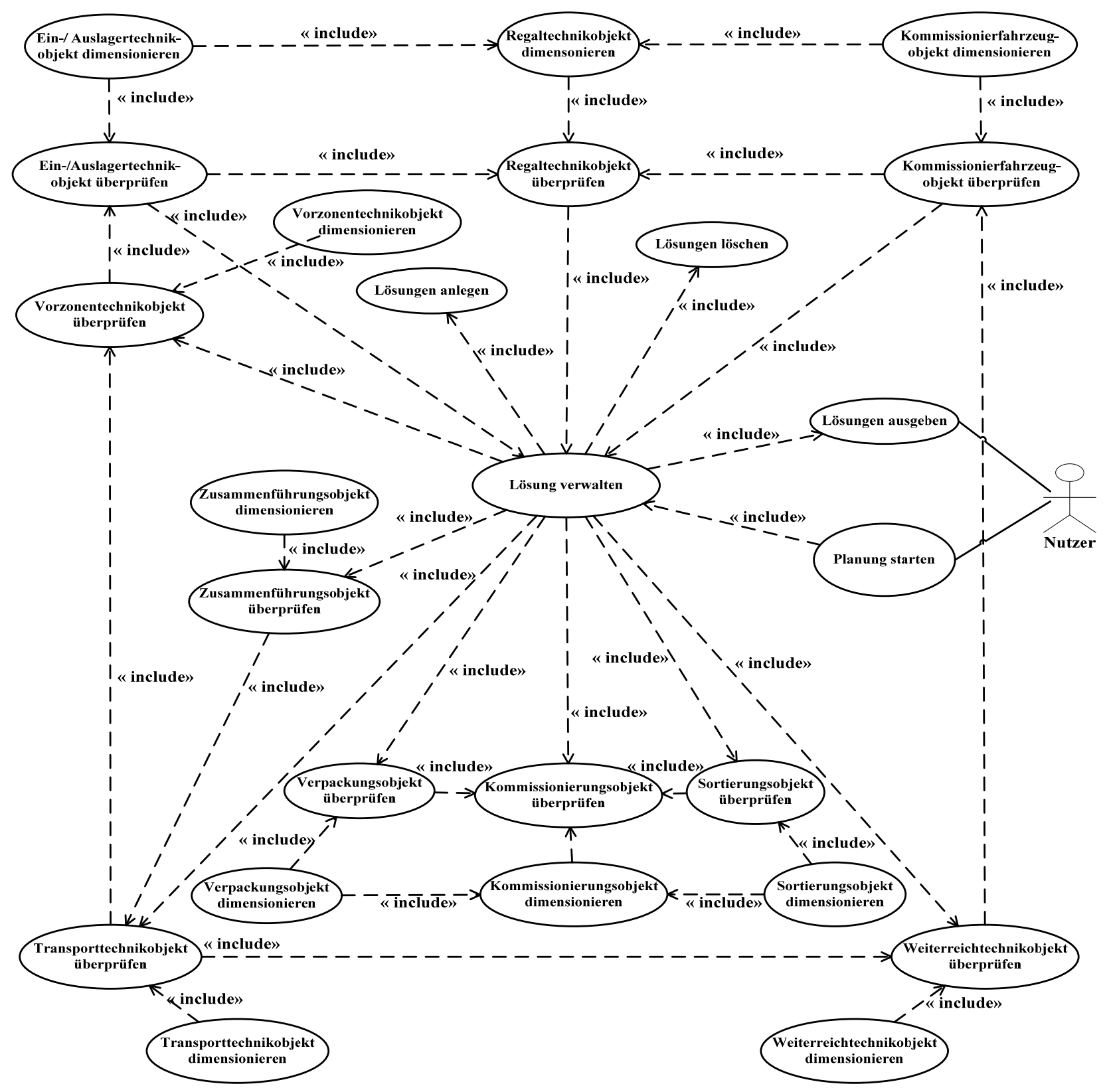

Abbildung 4. Domänenbeschreibungsdiagramm Planungsfunktionen

Abbildung 4 hingegen zeigt ein vereinfachtes Domänenbeschreibungsdiagramm für die systeminternen Funktionen. Es enthält die Funktionen, welche das System abbilden muss, um die Grobkonzepte für ein Kommissioniersystem automatisiert zu generieren. Beispielsweise muss das System ein Regaltechnikobjekt dimensionieren oder Lösungen verwalten können.

Nachfolgend werden die Entwicklung dieser Anwendungsfälle und der damit verbundene Systemaufbau näher erläutert.

\subsubsection{ENTWICKLUNG VON ANWENDUNGSFÄLLEN FÜR DIE SYSTEMISCHE PLANUNG}

Der erste Schritt zur Entwicklung dieser Anwendungsfälle war die Untersuchung der Funktionen für einen automatisierten den Konzeptentwurf. Dazu wurde zunächst die Aufgabenstellung der Grobplanung näher beleuchtet.

Während der Grobplanung eines Kommissioniersystems sind die Komponenten, aus denen das Kommissioniersystem bestehen soll, zu gestalten. Es stellt sich die Frage, was eine Komponente eines Kommissioniersystems ist und aus welchen es bestehen kann. Einige mögliche Komponenten sind in Abbildung 5 dargestellt. So be- 
steht beispielsweise ein klassisches Automatisches Kleinteilelager aus einer Behälterregaltechnik, Regalbediengeräten (Ein-/ Auslagertechnik) und einer Vorzone mit mind. einem Fördertechnikloop (Vorzonentechnik) und Kommissionierarbeitsplätzen (Kommissionierung).

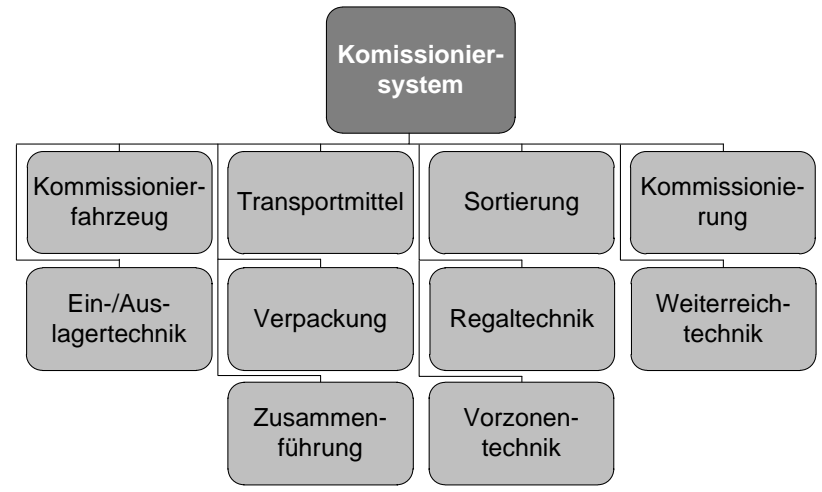

Abbildung 5. Komponenten eines Kommissioniersystems

Diese Detaillierungsebene ist jedoch für die Entwicklung von Grobkonzepten nicht ausreichend. In Grobkonzepten werden nicht die Komponenten betrachtet, sondern vielmehr die technische Ausprägung dieser Komponenten. Diese müssen folglich weiter spezifiziert werden, indem die technischen Realisierungsmöglichkeiten für jede Komponente identifiziert werden. Einige mögliche Realisierungen sind in Abbildung 6 beispielhaft für die Komponente Regaltechnik dargestellt. Die technische Realisierung Leer entspricht einer Bodenblocklagerung, also einer Lagerung ohne Regal.

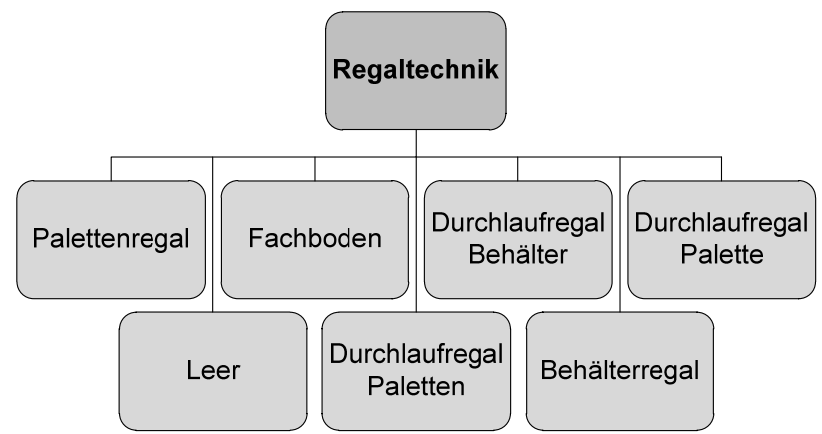

Abbildung 6. Technische Realisierung der Komponente Regaltechnik

Jede technische Realisierungsmöglichkeit der Komponenten wird im Planungssystem durch einen Agenten repräsentiert. Beispielsweise wird ein Fachboden-Agenten oder ein Behälterregal-Agenten implementiert. Dies ist in Abbildung 4 dadurch angedeutet, dass in den Anwendungsfällen bspw. nicht Lagertechnik dimensionieren, sondern Lagertechnikobjekt dimensionieren zu finden ist. Jeder Agent hat das Ziel in den Planungsprozess integriert zu sein. Er überprüft konstant, ob seine Eigenschaften mit den vom Nutzer gestellten Anforderungen und mit den Agenten anderer Komponenten kompatibel sind. Ist dies der Fall, kann er weiterhin an der Planung beteiligt sein. Ansonsten wird der Agent inaktiv.
Damit das Planungssystem eine Grobplanung durchführen kann, müssen für jede Komponente aus dem Pool von Agenten (technischen Realisierungen) die geeigneten Agenten ausgewählt und dimensioniert werden, wobei die Wechselwirkungen zwischen den Komponenten berücksichtigt werden müssen.

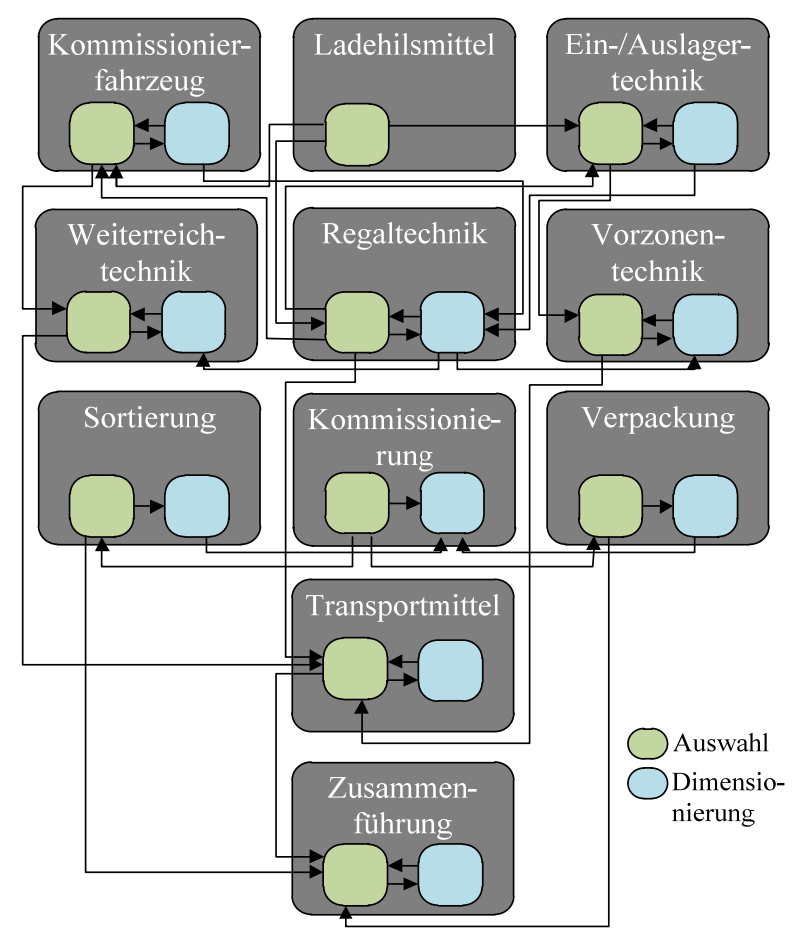

Abbildung 7. Beziehungen zwischen den Komponenten während der Planung

Zur Verdeutlichung der vielfältigen Wechselwirkungen zwischen den Komponenten sind in Abbildung 7 einige Beziehungen für die wesentlichen technischen Komponenten eines Kommissioniersystems dargestellt. Sie sind unterteilt nach den Gesichtspunkten Auswahl und Dimensionierung. Diese Beziehungen müssten in einem hierarchischen Planungsmodell von einer zentralen Instanz während der Planung berücksichtigt und verwaltet werden. In dem in diesem Beitrag beschriebenen Agentensystem, einem dezentralen Ansatz, sollen diese Beziehungen und Wechselwirkungen während des Planungsprozesses durch Interaktionen der entsprechenden Agenten abgebildet werden.

\subsubsection{BESCHREIBUNG DER SYSTEMFUNKTIONEN}

Ergänzend zu den Anwendungsfällen in den oben dargestellten Domänenbeschreibungsdiagrammen, ist es für ein umfassendes Verständnis sinnvoll, die Systemfunktionen in natürlicher Sprache zu beschreiben. Dies erfolgt in tabellarischer Form und ist angelehnt an die Beschreibungssystematik der objektorientierten Analyse, wie sie bei [Oes06] dargestellt ist. Für den Anwendungsfall Lösung ausgeben ist eine derartige Beschreibung in der Abbildung 8 aufgeführt. 


\begin{tabular}{|l|l|}
\hline Name & Lösung ausgeben \\
\hline $\begin{array}{l}\text { Kurz- } \\
\text { beschreibung }\end{array}$ & $\begin{array}{l}\text { Aufgabe dieser Funktion ist es, die } \\
\text { ermittelten Ergebnisse an den Nut- } \\
\text { zer auszugeben und visuell aufzu- } \\
\text { bereiten. Ausgegeben werden soll } \\
\text { ein Blockschaubild mit der Benen- } \\
\text { nung der Funktionsbereiche und } \\
\text { der eingesetzten Techniken und } \\
\text { Kosten. }\end{array}$ \\
\hline Akteure & Lösungsagenten \\
\hline Auslöser & Personalkosten wurden berechnet. \\
\hline Ergebnisse & $\begin{array}{l}\text { Ausgehende Daten sind für jede } \\
\text { Lösung die Blockschaubilder und } \\
\text { die markanten Eckpunkte. }\end{array}$ \\
\hline $\begin{array}{l}\text { Eingehende Da- } \\
\text { ten }\end{array}$ & $\begin{array}{l}\text { Informationen der Lösungsagenten } \\
\text { zu den Lösungsalternativen }\end{array}$ \\
\hline Vorbedingungen & Personalkosten wurden berechnet. \\
\hline Nachbedingungen & Keine \\
\hline Essenzielle & $\begin{array}{l}\text { 1. Blockschaubild generieren } \\
\text { 2. Markante Daten in den Blöcken } \\
\text { im Schaubild hinterlegen } \\
\text { 3. Die Blockschaubilder visualisie- } \\
\text { ren }\end{array}$ \\
\hline
\end{tabular}

Abbildung 8. Tabelle Beschreibung Systemfunktionen

\subsection{DETAILLIERUNG DER VORBEREITENDEN TÄTIGKEITEN}

Das in Abbildung 3 dargestellte Domänenbeschreibungsdiagramm für die vorbereitenden Tätigkeiten ist zur detaillierten Betrachtung der Systemfunktionen, aber auch zum Verständnis der Planungsvorgehensweise weiter zu spezifizieren. An dieser Stelle ist in der Abbildung 9 beispielhaft für den Anwendungsfall Restriktionen aufnehmen das Detaildomänenbeschreibungsdiagramm dargestellt.

Das System fragt vom Nutzer die für die Planung notwendigen Informationen ab. Die Restriktionen werden dabei in räumliche Restriktionen, Arbeitszeiten und Unternehmensrestriktionen (bspw. maximale Investitionssumme) differenziert. Zudem hat der Planer die Möglich- keit, technische Realisierungen oder (sofern zwischen technischen Realisierungen unterschiedlicher Anbieter unterschieden wird) die Techniken einzelner Anbieter auszuschließen. Dadurch kann der Nutzer bspw. die Generierung von Konzepten mit Fachbodenanlagen unterbinden. Diese Informationen werden vom System gespeichert und stehen den Agenten während der Planung zur Verfügung.

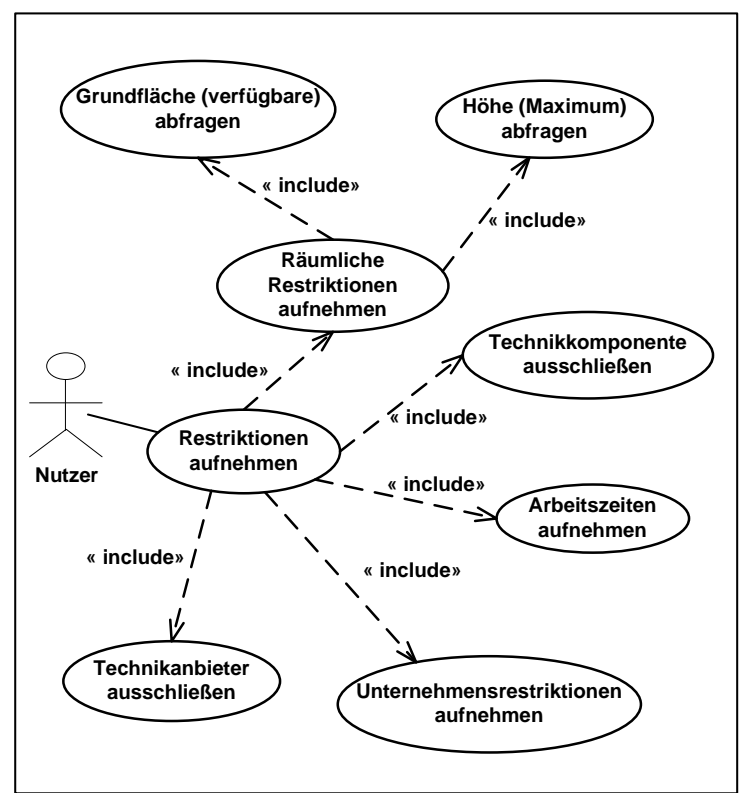

Abbildung 9. Detaildomänenbeschreibungsdiagramm Restriktionen aufnehmen

\subsection{Detaillierung Der Systemischen PlanUng}

Die Prinzipien der systemischen Planung wurden in der Abbildung 4 dargestellt. Diese Anwendungsfälle müssen präzisiert werden, um die Planungsvorgehensweise und die Funktionalität des Planungssystems evaluieren und nachvollziehen zu können. Beispielhaft ist die Beschreibung des Anwendungsfalles Regaltechnikobjekt überprüfen in der Abbildung 10 als vereinfachter Prozess dargestellt. Dieser Prozess entspricht der Auswahl der Regaltechnikobjekte für die gegebenen Nutzeranforderungen. Er wird von jedem Agenten (der eine technische Realisierung der Regaltechnik repräsentiert) für jeden Funktionsbereich durchlaufen.

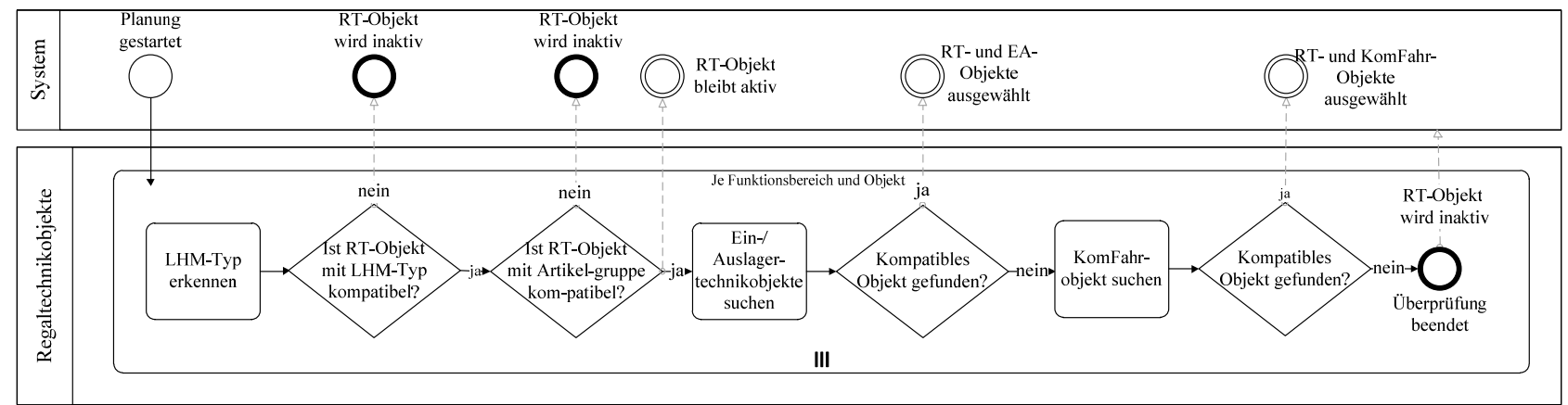

Abbildung 10. Prozess der Auswahl der technischen Realisierungen für die Komponente Regaltechnik 
Als Beispiel sei hier ein Funktionsbereich für die Lagerung und Kommissionierung von Paletten genannt. Sobald die Planung vom Nutzer gestartet wurde, werden alle Regaltechnikagenten anfangen, zu überprüfen, ob sie für die Lagerung von Paletten eingesetzt werden können. Alle Agenten, die eine Behälterlagertechnik repräsentieren, werden folglich inaktiv. Die Agenten, welche die Palettenlagertechnik repräsentieren, verbleiben aktiv im System. Sie können mit den Agenten in Kontakt treten, die eine Ein-/Auslagertechnik (z.B. RGB) oder Kommissionierfahrzeuge (bspw. Kommissionierstapler) repräsentieren.

Im Ergebnis bleiben im Planungssystem die Agenten aktiv, welche die Anforderungen prinzipiell erfüllen können. Diese Agenten gehen in den Austausch mit den Agenten anderer Komponenten und generieren mit denen (sofern sie kompatibel sind) Lösungsalternativen. Diese Lösungen werden vom System verwaltet. Nach der Berechnung der Investitions- und Personalkosten können dem Nutzer verschiedene Lösungskonzepte für sein neues Kommissioniersystem angezeigt werden. Aus diesen kann der Anwender die für ihn optimale Alternative auswählen.

\section{ZuSAMMENFASSUNG UND AUSBLICK}

Die Planung intralogistischer Systeme und insbesondere von Kommissioniersystemen stellt die Praxis tagtäglich vor große Herausforderungen. Die Planungsdauer ist zu lang. Die Planung kann nur von wenigen Experten effizient und in hoher Qualität ausgeführt werden. Die eingesetzten Methoden sind zu starr, um flexibel und schnell auf Veränderungen der Planungssituation zu reagieren.

Diese Schwächen liegen zum einen in der Komplexität der Planungsaufgabe. Zum anderen ist die Planung bis heute ein manueller Prozess, da eine effektive und anwendbare Computerunterstützung des Planers fehlt. Ein rechnerbasiertes Assistenzsystem konnte bisher aufgrund des vorherrschenden Planungsansatzes des hierarischen, top-down orientierten Planungsvorgehens nicht entwickelt werden. Die Problemstellung der Planung eines Kommissioniersystems ist zu komplex, um diese mit einem hierarischen oder zentralen System abbilden zu können. Es ist an der Zeit, einen anderen Weg zu gehen. Dezentrale Systeme sind nicht nur für die Steuerung, sondern auch für die Planung von Intralogistiksystemen interessant.

Daher wurde am Fraunhofer-Institut für Materialfluss und Logistik IML eine Forschungsinitiative initiiert, welche die Erforschung und Entwicklung der automatisierten Planung von Intralogistiksystemen zum Ziel hat. Teil dieser Initiative ist die Entwicklung eines agentenbasierten Planungsmodells für die Grobplanung von Kommissioniersystemen. Auszüge des Anforderungsmodells für dieses Planungssystem wurden in diesem Beitrag vorgestellt.
Die nächsten Schritte liegen in der Identifikation der Agenten und deren Rollen innerhalb des Systems. Der Prozessablauf innerhalb des Systems zur Entwicklung der Lösungsalternativen ist ausführlich und im Detail zu untersuchen. Damit verbunden sind die Art der Kommunikation zwischen den Agenten und die Inhalte der Nachrichten zu analysieren und zu dokumentieren.

\section{LITERATUR}

[AMW10] Apple, Jr.; James, M.; Meller, Russel D.; White, John A. Jr.: Empericallybased warehouse design: Can academics accept such an approach? In: Progress in material handling research, 2010

[CCS04] Chella, Antonio; Cossentino, Massimo; Sabatucci, Luca: Tools and Patterns in Designing Multi-Agent Systems with PASSI. WSEAS Transactions on Communications, 3, 2004

[CP02] Cossentino, Massimo; Potts, Colin: PASSI: a Process for Specifying and Implementing Multi-Agent Systems Using UML. 2002

[DHR+05] Davidsson, Paul; Henesy, Lawrence; Ramstedt, Linda et al.: An analysis of agent-based approaches to transport logistics. In: Transportation Research. Part C, 2005, S. 255-271

[FO05]

Fujita, K; Ohtani, S.: Hierarchical Contexts for Emergent Layout Design Synthesis: Preliminary Development of Agent-Based Plant Layout Design System. In: Journal of Manufacturing Systems, Vol. 34, 2005, S. 161-170

[GGU09] Günthner, Willibald A.; Galka, Stefan; Ulbrich, Alexander: Neue Werkzeuge für die Planung von Kommissioniersystemen. In: Jahrbuch der Logistik, 2009, S. 285-289

[GH10] Güntner, Willibald A.; ten Hompel, Michael (Hrsg.): Internet der Dinge in der Intralogistik. Berlin/Heidelberg; Springer-Verlag, 2010

[Gud10] Gudehus, Timm: Logistik. Berlin/Heidelberg: Springer Verlag, 2010

[HB10] Hellingrath, Bernd; Böhle, Carsten: Integrierte agentenbasierte Produktionsund Logistikplanung in der Supply 
Chain. In: Künstliche Intelligenz, Jg. 24, 2010, S. 115-122

[Hom08] ten Hompel, Michael: Intralogistik auf dem Weg vom Prozess zum Service. In Baumgarten, Helmut (Hrsg.): Das Beste der Logistik. Innovationen, Strategien, Umsetzung. Berlin/Heidelberg: Springer-Verlag, 2008

[HSB11] ten Hompel, Michael; Sadowsky, Volker; Beck, Maria: Kommissionierung. Berlin/Heidelberg: Springer-Verlag, 2011

[JD89] Jünemann, Reinhardt; Daum, Matthias: Materialfluß und Logistik. Berlin/ Heidelberg: Springer Verlag, 1989

[JNS09] Jobi, Boris; Neuhäuser, Daniel; Sommer, Tobias: Ganzheitliche Planung logistischer Systeme - Hilfestellung durch EDV gestützte Assistenzsysteme. In: Wolf-Michael Scheid. 5. Fachkolloquium der Wissenschaftlichen Gesellschaft für Technische Logistik. Ilmenau: Univ.-Verl. Ilmenau, 2009

[JT12] Jodin, Dirk; Trummer, Wolfgang: Zeitaufwand reduzieren - Wissensbasierte Methoden zur Planung von intralogistischen Anlagen und Systemen. In: Fördern und Heben Projektguide Intralogistik, 2012, S. 10-13

[KSN+11] Kamagaew, Andreas; Stenzel, Jonas; Nettsträter, Andreas: ten Hompel, Michael: Concept of Cellular Transport Systems in Facility Logistics. In: ICARA 2011, 5th IEEE International Conference on Automation, Robotics and Applications. Wellington, Neuseeland

[LCL10] Libert, Sergey; Chisu, Razvan; Luft, Arthur: Softwarearchitektur für eine agentenbasierte Materialflusssteuerung. In Günthner, Willibald; ten Hompel, Michael (Hrsg.): Internet der Dinge in der Intralogistik. Berlin/Heidelberg: Springer-Verlag, 2010

[Lib11] Libert, Sergey: Beitrag zur agentenbasierten Gestaltung von Materialflusssteuerungen. Dortmund: Dissertation, 2011

[MM07] Meinhardt, Ingolf; Marquardt, HansGeorg: Offenes Baukastensystem zur effizienten Dimensionierung von Materialflusssystemen. In: Logistics Journal. Nicht referierte Veröffentlichungen ISSN 1860-5923, 2007

[Oes06] Oestereich, Bernd: Objektorientierte Softwareentwicklung - Analyse und Design mit der UML. München: Oldenbourg Wissenschaftsverlag, 2006

[Par98] Paranuk, H. van Dyke (Hrsg.): Practical and Industrail Applications of AgentBased Systems. Environmental Research Institut of Michigan (ERIM), 1998

[Pot94] Potyka, Sebastian: Systematik zur Selektion von Kommissioniersystemen in der Planung. Dortmund: Dissertation, 1994

[Sad07] Sadowsky, Volker: Beitrag zur analytischen Leistungsermittlung von Kommissioniersystemen. Dortmund: Dissertation, 2007

[Sch08] Scholl, Armin: Modellierung logistischer Systeme. In: Arnold, Dieter; Furmans, Kai; Isermann, Heinz, et. Al. (Hrsg.): Handbuch Logistik. Berlin/Heidelberg: Springer-Verlag, 2008, S. 35-43.

[Töp95] Töpper, Hans-Henning: Entwicklung eines Verfahrens zur Auswahl und technischen Gestaltung von Kommissioniersystemen. Dortmund: Dissertation, 1995

[Ulb10] Ulbrich, Alexander Stephan: Simulationsgestützte Grobplanung von Kommissioniersystemen. München: Dissertation, 2010

[VDI3590] Verein Deutscher Ingenieure (Hrsg.): VDI 3590 Blatt 1 Kommissioniersysteme - Grundlagen. Berlin, Beuth- Verlag, 1994 